\title{
Focused correlation, confirmation, and the jigsaw puzzle of variable evidence
}

\author{
Maximilian Schlosshauer and Gregory Wheeler \\ Forthcoming in Philosophy of Science
}

\begin{abstract}
Focused correlation compares the degree of association within an evidence set to the degree of association in that evidence set given that some hypothesis is true. A difference between the confirmation lent to a hypothesis by one evidence set and the confirmation lent to that hypothesis by another evidence set is robustly tracked by a difference in focused correlations of those evidence sets on that hypothesis, provided that all the individual pieces of evidence are equally, positively relevant to that hypothesis (Wheeler and Scheines 2010). If focused correlation is interpreted as a 'coherence' measure, this result is theoretically significant because it establishes conditions under which a difference in coherence entails a difference in confirmation. However, the result is of limited practical benefit because the equal relevance condition is a very strong restriction. In this essay we prove tracking results for focused correlation analogous to Wheeler and Scheines's results but for cases involving unequal relevance. Our result is robust as well, and we retain conditions for bidirectional tracking between incremental confirmation measures and focused correlation. The cases in which tracking fail are instructive, suggesting that focused correlation is a stronger measure of evidential support than classical incremental confirmation measures. We illustrate this point with examples, situate our results in relationship to the noted impossibility results concerning Bayesian epistemic coherence measures, and offer an additional reason for distinguishing classical, incremental confirmation from other notions of confirmation.
\end{abstract}

\section{Introduction}

Auditors have found an irregularity in the books for Acme, Inc. Bungled paperwork explains most anomalies of this kind, but fraud cannot be ruled out, so teams are assembled to investigate. Team One learns that Carson, a longtime Acme bookkeeper, is divorcing his wife, which is sad news for the Carson family, but of little interest to Team One. Most people manage a divorce without defrauding their employer, after all. Team Two learns that Carson has a Brazilian lover, but they merely pause to think, lucky Carson. Team Three finds out that Carson took a flight to Rio de Janeiro seated beside a stunning but dodgy Brazilian financier.

Considered in isolation, each team's evidence signals little more than middle-life boredom. Yet when taken together the evidence suggests fraud. It's not the facts alone 
that warrant hauling in Carson for questioning, but rather how those pieces of evidence fit together into a compelling case against him.

But what does it mean for evidence to 'fit together,' and what is it about combined evidence that lends more support to a hypothesis than each piece of evidence on its own? One answer is to compare the likelihood of evidence occurring together to its co-occurrence given a cause or some unifying explanation. The events of the story are unusual on their own, but a conspiracy to defraud Acme involving Carson provides a unifying explanation for those events, one that would be alarming enough for Acme investigators to want a word with Mr. Carson.

The core idea animating this example is encapsulated in a measure called focused correlation (Myrvold 1996, Wheeler 2009), which is defined by Equation (1) for $n$ binary evidence variables and a single binary hypothesis variable.

$$
\begin{aligned}
\operatorname{For}_{\boldsymbol{H}}\left(\boldsymbol{E}_{1}, \ldots, \boldsymbol{E}_{n}\right)=\frac{P\left(\boldsymbol{H} \mid \boldsymbol{E}_{1} \cap \cdots \cap \boldsymbol{E}_{n}\right) P(\boldsymbol{H})^{n-1}}{P\left(\boldsymbol{H} \mid \boldsymbol{E}_{1}\right) \times \cdots \times P\left(\boldsymbol{H} \mid \boldsymbol{E}_{n}\right)} \\
\quad=\frac{P\left(\boldsymbol{E}_{1} \cap \cdots \cap \boldsymbol{E}_{n} \mid \boldsymbol{H}\right) / P\left(\boldsymbol{E}_{1} \mid \boldsymbol{H}\right) \times \cdots \times P\left(\boldsymbol{E}_{n} \mid \boldsymbol{H}\right)}{P\left(\boldsymbol{E}_{1} \cap \cdots \cap \boldsymbol{E}_{n}\right) / P\left(\boldsymbol{E}_{1}\right) \times \cdots \times P\left(\boldsymbol{E}_{n}\right)} .
\end{aligned}
$$

Focused correlation has been given a variety of interpretations: to capture the effect that diversity of evidence has upon confirmation of some hypothesis (Myrvold 1996); to provide a partial explication of the relationship between epistemic coherence and incremental confirmation of a hypothesis (Wheeler 2009); and, in normalized form, to provide an account of unified evidence for a hypothesis (Myrvold 2003). But, the basic idea is that by comparing the degree of association within an evidence set to the degree of association in that evidence set given some hypothesis, in certain circumstances several questions about confirmation relationships between evidence and that hypothesis can be answered.

The conditions under which focused correlation tracks confirmation, pace Myrvold (2003), depend on what type of logical or causal structure governs the relationships between evidence and hypothesis in a problem (Wheeler and Scheines 2010). For example, Erik Olsson (2005) and Luc Bovens and Stephan Hartmann (2006) have drawn attention to problematic features of models in which all binary evidence variables $\boldsymbol{E}_{i}$ of a problem are conditionally independent of the binary hypothesis variable $\boldsymbol{H}$, i.e., $\forall i \neq j, \boldsymbol{E}_{i} \Perp \boldsymbol{E}_{j} \mid \boldsymbol{H}$. The correlation between any distinct pair of evidence variables $\boldsymbol{E}_{i}$ and $\boldsymbol{E}_{j}$ in this class of models is the product of the correlations between each $\boldsymbol{E}$ and $\boldsymbol{H}$ (Danks and Glymour 2001). But this fact yields counter-intuitive results if one interprets association as epistemic coherence, since then association among the evidence is strictly less than the (non-deterministic) confirmation that each piece of evidence lends to the hypothesis.

In the case of focused correlation, it is easy to see from Equation (1) that the focused correlation of an evidence set with respect to some hypothesis under this conditional independence assumption is strictly less than one, thus is negative, whereas the confirmation of the hypothesis by that evidence may be positive. This conditional independence condition is central to the witness testimony models of (Olsson 2005) and 
(Bovens and Hartmann 2003), and it is the condition that drives their noted negative results concerning the construction of a probabilistic measure of coherence (Wheeler 2009).

But, contra Olsson (2005, p. 135), there are interesting cases outside of this class of models where a measure like focused correlation can be useful and, pace Luc Bovens and Stephan Hartmann (2006), there are conditions under which a difference in confirmation lent to some hypothesis by two evidence sets is tracked by a difference in their focused correlation, even within this obstinate class of models. ${ }^{1}$ To illustrate, consider again L'affaire de Carson. Suppose that a performance review of the Acme Investigation Department involves comparing the evidence set $\mathbf{E}$, consisting of a divorcing employee who traveled with a dubious associate, to the evidence set $\mathbf{E}^{*}$, consisting of a divorcing employee who is having an affair. Acme is a big company with many cases on file, and their models suggest that $\mathbf{E}$ is a better indicator of fraud than $\mathbf{E}^{*}$ because fraud is a slightly 'better' explanation for the co-occurrence of divorce and suspicious travel than it is for divorce and adulterous affair. Question: under what conditions can Acme management draw this inference?

In (Wheeler and Scheines 2010) sufficient conditions for a wide variety of incremental confirmation measures are given under which a difference in focused correlation entails a difference in confirmation lent to that hypothesis and vice versa. But their results are only trivially true in this problematic class of models, because one of the conditions for the comparison result is that all individual items of evidence have equal relevance to the hypothesis. In effect this restrictive condition, when combined with the conditional independence assumption mentioned above, deals with the problematic cases driving Bovens and Hartmann's counter-examples (Bovens and Hartmann 2003, 2006) by ensuring that at least one of the antecedent conditions is false. The motivation for this strong assumption is clear in their project, for they address whether a difference in association of two evidence sets induced by some hypothesis can track confirmation of that hypothesis. But including equal relevance as a ceteris paribus condition won't do for practical comparisons of evidence sets; it is much too restrictive.

In this paper we show the equal evidence assumption can indeed be relaxed to identifiable cases involving unequal relevance. Our result is robust as well, and we retain conditions for bidirectional tracking between the class of incremental confirmation measures and focused correlation. The cases in which tracking fail are instructive, suggesting that focused correlation is a stronger measure of evidential support than classical incremental confirmation measures, and we illustrate this point through some examples.

The structure of the paper is as follows. In Section 2 we set up the machinery by making explicit our notation and the confirmation measures we consider. Section 3 presents our results, and in Section 4 we explain why two common non-incremental

\footnotetext{
${ }^{1}$ Observe that this problematic class of models may be characterized by those in which this conditional independence condition holds. Hence, that class of models-provided that the assumptions needed to activate the theory of graphical causal models are satisfied - can be identified non-parametrically and handled by other means. So, while true that no probabilistic measure of coherence can operate correctly within this class, that's only one way to look at it; another is to observe that there is no need for a coherence measure in this class of models.
} 
confirmation measures are not tracked by focused correlation. This analysis offers further evidence for distinguishing between classical, incremental confirmation measures, and other notions of confirmation. Proofs are set in an appendix.

\section{Setup}

In this section we specify notation and list the confirmation functions we will consider. Define a probability space $(\Omega, \mathcal{F}, P)$ such that $\mathcal{F}$ is a $\sigma$-algebra over a set $\Omega$ and $P: \mathcal{F} \longrightarrow[0,1]$ is a probability measure defined on the space $(\Omega, \mathcal{F}, P)$ satisfying

P1. $P(\Omega)=1$

P2. $P\left(\bigcup_{i=1}^{\infty} X_{i}\right)=\sum_{i=i}^{\infty} P\left(X_{i}\right)$, when $X_{i}$ are countable, pairwise disjoint elements of $\mathcal{F}$.

A probability structure is a tuple $M=(\Omega, \mathcal{F}, P, V)$, where $(\Omega, \mathcal{F}, P)$ is a probability space and $V$ is an interpretation function associating each element $\omega \in \Omega$ with a truth assignment on the primitive propositions in $A, B, \ldots \in \Phi$ such that $V(\omega)(A) \in\{1,0\}$ for each $\omega \in \Omega$ and for every proposition in $\Phi$.

For each primitive proposition in $\Phi$, we define $M, \omega=A$ iff $V(\omega)(A)=1$ and proceed by induction on the structure of propositional formulas. Since $P$ is defined on events in $\mathcal{F}$ rather than propositions, let $\llbracket A \rrbracket_{M}$ denote the set of outcomes within $\Omega$ in $M$ where $A$ is true, which will correspond to a subset of $\mathcal{F}$. The following makes explicit the relationship between propositions and events for arbitrary propositional formulas $A$ and $B$ :

$$
\begin{aligned}
& \text { i. } \llbracket A \wedge B \rrbracket_{M}=\llbracket A \rrbracket_{M} \cap \llbracket B \rrbracket_{M}, \\
& \text { ii. } \llbracket A \vee B \rrbracket_{M}=\llbracket A \rrbracket_{M} \cup \llbracket B \rrbracket_{M}, \\
& \text { iii. } \llbracket \neg A \rrbracket_{M}=\overline{\llbracket A \rrbracket_{M}} .
\end{aligned}
$$

An evidence set is a set of propositions, written $\mathbf{E}=\left\{E_{1}, E_{2}\right\}$. We defined focused correlation in Equation (1) with respect to random variables $\boldsymbol{E}_{i}$, and $\boldsymbol{H}$, each taking values 0 or 1 . We use the abbreviation $E$ for $\boldsymbol{E}=1$ and $\neg E$ for $\boldsymbol{E}=0$, and likewise will use the abbreviation $P\left(E_{1} \wedge \neg E_{2} \mid H\right)$ for $P\left(\boldsymbol{E}_{1}=1 \cap \boldsymbol{E}_{2}=0 \mid \boldsymbol{H}=1\right)$. We will relax notation by expressing focused correlation of propositions as well as of variables, using the conventions here for distinguishing between propositions and variables to signal which is which.

\subsection{Confirmation measures}

A statement $E$ is confirmation to hypothesis $H$ with respect to a classical probability model specifying the measure $P$ just when $E$ and $H$ are positively correlated under $P$, 
that is when $P(H \mid E)>P(H)$. A confirmation function measures the degree to which evidence confirms a hypothesis, and there are several proposals. ${ }^{2}$

$$
\begin{aligned}
i n c_{1}\left(H, E_{1}, E_{2}\right) & :=P\left(H \mid E_{1} \wedge E_{2}\right)-P\left(H \mid E_{1}\right) \\
i n c_{2}\left(H, E_{1}, E_{2}\right) & :=\frac{P\left(H \mid E_{1} \wedge E_{2}\right)-P\left(H \mid E_{1}\right)}{1-P\left(H \mid E_{1}\right)} \\
k o(H, \mathbf{E}) & :=\frac{P(\mathbf{E} \mid H)-P(\mathbf{E} \mid \neg H)}{P(\mathbf{E} \mid H)+P(\mathbf{E} \mid \neg H)} \\
r(H, \mathbf{E}) & :=\log \frac{P(H \mid \mathbf{E})}{P(H)} \\
l(H, \mathbf{E}) & :=\log \frac{P(\mathbf{E} \mid H)}{P(\mathbf{E} \mid \neg H)}
\end{aligned}
$$

\subsection{Comparing equal-relevance evidence sets}

In our discussion throughout, we assume that $P(\mathbf{D})$, a probability distribution defined over a domain of propositions $\langle H, \mathbf{E}\rangle$, is positive. In addition, we may appeal to two conditions.

(A1) Positive Relevance: all propositions in $\mathbf{E}$ are positively relevant to $H$ just in case, $\forall E_{i} \in \mathbf{E}, P\left(H \mid E_{i}\right)>P(H)>P\left(H \mid \neg E_{i}\right)$.

(A2) Equal Relevance: all propositions in a set of evidence $\mathbf{E}$ are equally confirmatory just in case $\forall E_{i}, E_{j} \in \mathbf{E}, P\left(H \mid E_{i}\right)=P\left(H \mid E_{j}\right)$, and $P\left(H \mid \neg E_{i}\right)=P\left(H \mid \neg E_{j}\right)$.

First, evidence and hypothesis may be correlated or anti-correlated, unless independent. Thus evidence may be either positively relevant or negatively relevant to a hypothesis, if it is relevant at all. Condition (A1) restricts attention to cases where evidence is positively relevant. Second, strength of relevance need not be the same, but condition (A2) restricts attention to just those cases where all pieces of evidence are equally relevant to the hypothesis. ${ }^{3}$ This is the condition we shall explore how to weaken. But first, let's see the benefit from a positive distribution over $\mathbf{D}$ that satisfies both (A1) and (A2).

\footnotetext{
${ }^{2}$ inc $_{1}$ and $i n c_{2}$ are both variants of L. Jonathan Cohen's notion of incremental convergence (Cohen 1977): $i n c_{1}$ reports the contribution that $E_{2}$ simpliciter makes to $H$, whereas $i n c_{2}$ reports the contribution $E_{2}$ makes with respect to the possible available evidence; $i n c_{1}$ and $i n c_{2}$ are the two cases that comprise measure $Z$ (Crupi et al. 2007), where $i n c_{2}(H, E)$ is used if $P(H \mid E)>P(H)$, inc $1(H, E)$ otherwise. $k o$ is the Kemeny and Oppenheim fitness measure (Kemeny and Oppenheim 1952). $r$ is a generic relevance measure, versions of which have been endorsed from (Keynes 1921) to (Milne 1997), among others. $l$ is ordinally equivalent to $k o$. $k o, r, l$ are typically discussed for evidence $E$ representing an evidence set $\mathbf{E}$ of arbitrary size by a single conjunction of the propositions in $\mathbf{E}$. We will discuss cases where $|\mathbf{E}|=2$. See (Kyburg 1983) for an overview of confirmation measures, and (Eells and Fitelson 2002) for a recent discussion.

${ }^{3}$ See Lemma 4.2 and remarks in the Appendix about the strength of these two assumptions when combined.
} 
Proposition 2.1. (Wheeler and Scheines, 2010) If $\mathbf{E}=\left\{E_{1}, E_{2}\right\}$ and $\mathbf{E}^{*}=$ $\left\{E_{1}, E_{3}\right\}$, and $\mathbf{E} \cup \mathbf{E}^{*}$ satisfies (A1) and (A2) with respect to $H$, then all the following inequalities are equivalent: ${ }^{4}$

- $\operatorname{For}_{H}(\mathbf{E})>\operatorname{For}_{H}\left(\mathbf{E}^{*}\right)$

- $r(H, \mathbf{E})>r\left(H, \mathbf{E}^{*}\right)$

- $l(H, \mathbf{E})>l\left(H, \mathbf{E}^{*}\right)$

- $k o(H, \mathbf{E})>k o\left(H, \mathbf{E}^{*}\right)$

- $i n c_{1}(H, \mathbf{E})>i n c_{1}\left(H, \mathbf{E}^{*}\right)$

- $i n c_{2}(H, \mathbf{E})>i n c_{2}\left(H, \mathbf{E}^{*}\right)$

Proposition 2.1 tells us that focused correlation tracks incremental confirmation and vice versa, but the proof leans on (A2) to isolate the effect of focused correlation on incremental confirmation. This condition is too restrictive to exploit in an application and, as observed in the Introduction, it deals with a problematic class of models by effectively excluding them. In the next section we consider how to preserve these tracking properties of focused correlation without condition (A2).

\section{Unequal Relevance}

We consider, as before, two evidence sets $\mathbf{E}=\left\{E_{1}, E_{2}\right\}$ and $\mathbf{E}^{*}=\left\{E_{1}, E_{3}\right\}$, but now we shall relax assumption (A2): we no longer require that all propositions $E_{i}, i=1,2,3$, are equally confirmatory. That is, we allow for the degree of positive relevance $P\left(H \mid E_{i}\right)$ of the individual pieces of evidence to vary.

What we would like to check now is whether, in this generalized situation, focused correlation continues to track confirmation, and vice versa. That is, we would like to explore under what conditions Proposition 2.1 still holds. 'Confirmation' is here understood as quantified by the measures defined in Sec. 2.1. We shall denote these measures collectively by $c(H, \mathbf{E})$ in the following, i.e., $c(H, \mathbf{E})$ jointly stands for any of the functions $i n c_{1}(H, \mathbf{E}), i n c_{2}(H, \mathbf{E}), k o(H, \mathbf{E}), r(H, \mathbf{E})$, and $l(H, \mathbf{E})$.

A quick look at the definitions of these confirmation measures (Sec. 2.1) shows that they are based only on the probabilities of $H$ conditional on the full evidence set and on the shared evidence piece $E_{1}$, but not on the probabilities of $H$ conditional on the distinct individual pieces $E_{2}$ and $E_{3}$, i.e., not on $P\left(H \mid E_{2}\right)$ and $P\left(H \mid E_{3}\right)$. The appearance of $P\left(H \mid E_{2}\right)$ and $P\left(H \mid E_{3}\right)$ in Equation (1) is therefore a particular feature of focused correlation. This feature exemplifies the additional input to the measure, which takes into account the conditional probabilities $P\left(H \mid E_{i}\right)$ for all $E_{i} \in \mathbf{E}$.

\footnotetext{
${ }^{4}$ For the proposition to hold for $i n c_{1}$, we stipulate that $i n c_{1}(H, \mathbf{E})>\operatorname{inc} c_{1}\left(H, \mathbf{E}^{*}\right)$ stands for $i n c_{1}\left(H, E_{1}, E_{2}\right)>i n c_{1}\left(H, E_{1}, E_{3}\right)$. A similar remark applies for interpreting $i n c_{2}$.
} 
As an aside, we note that the relevance $P\left(H \mid E_{1}\right)$ of the evidence $E_{1}$ will not enter into the following considerations, not withstanding its role in $i n c_{1}$ and $i n c_{2}$. This is so because $E_{1}$ is shared among both evidence sets $\mathbf{E}$ and $\mathbf{E}^{*}$, and we are here concerned only with a comparison of the degrees of focused correlation and confirmatory power of these two sets.

\subsection{From focused correlation to confirmation}

Let us first describe the condition under which a larger degree of focused correlation for evidence set $\left\{E_{1}, E_{2}\right\}$ than for $\left\{E_{1}, E_{3}\right\}$ implies larger confirmation of the hypothesis by $\left\{E_{1}, E_{2}\right\}$ than by $\left\{E_{1}, E_{3}\right\}$.

Proposition 3.1. If $\operatorname{For}_{H}\left(E_{1}, E_{2}\right)>\operatorname{For}_{H}\left(E_{1}, E_{3}\right)$ and

$$
\frac{P\left(H \mid E_{2}\right)}{P\left(H \mid E_{3}\right)}>\frac{\operatorname{For}_{H}\left(E_{1}, E_{3}\right)}{\operatorname{For}_{H}\left(E_{1}, E_{2}\right)}
$$

then also $c\left(H,\left\{E_{1}, E_{2}\right\}\right)>c\left(H,\left\{E_{1}, E_{3}\right\}\right)$.

Thus the confirmation measures $c\left(H,\left\{E_{1}, E_{2}\right\}\right)$ track focused correlation if the bound (2) is fulfilled. In particular, this will be the case whenever $P\left(H \mid E_{2}\right) \geq P\left(H \mid E_{3}\right)$, but the implication $\operatorname{For}_{H}\left(E_{1}, E_{2}\right)>\operatorname{For}_{H}\left(E_{1}, E_{3}\right) \Rightarrow c\left(H,\left\{E_{1}, E_{2}\right\}\right)>c\left(H,\left\{E_{1}, E_{3}\right\}\right)$ will also hold if $P\left(H \mid E_{2}\right)<P\left(H \mid E_{3}\right)$ by an amount that is a function of the difference in the focused correlations of the two sets $\mathbf{E}$ and $\mathbf{E}^{*}$, as specified by (2). However, if $P\left(H \mid E_{2}\right)$ drops below this bound, then the tracking fails, and the confirmation measures $c\left(H,\left\{E_{1}, E_{2}\right\}\right)$ will indicate less confirmatory power for $\mathbf{E}$ than $\mathbf{E}^{*}$ despite the fact that $\mathbf{E}$ has larger focused correlation than $\mathbf{E}^{*}$.

This observation has an intuitive explanation, as $c\left(H,\left\{E_{1}, E_{2}\right\}\right)<c\left(H,\left\{E_{1}, E_{3}\right\}\right)$ is equivalent to $P\left(H \mid E_{1} \wedge E_{2}\right)<P\left(H \mid E_{1} \wedge E_{3}\right)$ when both (A1) and (A2) hold. Now, as mentioned above, focused correlation goes a step further than the measures $c\left(H,\left\{E_{1}, E_{2}\right\}\right)$, because it weighs the joint relevance $P(H \mid \mathbf{E})$ by how much each individual piece of evidence confirms $H$. If $E_{1}$ and $E_{2}$ individually confirm $H$ very little compared to the confirmation of $H$ provided by the conjunction $E_{1} \wedge E_{2}$, while the probability of $H$ given $E_{1} \wedge E_{3}$ does not exceed by much the product of the probabilities of $H$ given $E_{1}$ and $E_{3}$ alone, then the set $\left\{E_{1}, E_{2}\right\}$ may have larger focused correlation than the set $\left\{E_{1}, E_{3}\right\}$ even if $P\left(H \mid E_{1} \wedge E_{2}\right)<P\left(H \mid E_{1} \wedge E_{3}\right)$. In other words, $\operatorname{For}_{H}\left(E_{1}, E_{2}\right) \geq \operatorname{For}_{H}\left(E_{1}, E_{3}\right)$ may arise from a situation in which $P\left(H \mid E_{2}\right)$ is so much smaller than $P\left(H \mid E_{3}\right)$ as to be able to counteract the greater confirmatory power of $\mathbf{E}^{*}$ over $\mathbf{E}$ as quantified by the measures $c\left(H,\left\{E_{1}, E_{2}\right\}\right)$.

We will illustrate our results by presenting alternative versions of Mr. Carson's scandal. In each of our examples, we shall let $E_{1}$ represent the fact that Carson is divorcing his wife, $E_{2}=$ Carson's trip abroad seated next to a shady financier, $E_{3}=$ Carson's affair with a Brazilian lover, and $H=$ Carson swindled Acme. We begin by considering the case in which the confirmation measures $c(H, \mathbf{E})$ fail to track focused correlation. 
Example 1. Suppose that the evidence set $\left\{E_{1}=\right.$ divorce, $E_{2}=$ travel $\}$ has larger focused correlation than $\left\{E_{1}=\right.$ divorce, $E_{3}=$ affair $\}$, with respect to the hypothesis $H$. We interpret this relation as saying that evidence of both Carson's divorce proceedings and his travel cohere better toward supporting the hypothesis of fraud than evidence of Carson's divorce and affair.

But this fact does not necessarily mean that the coincidence of divorce and travel is also more confirmatory of the hypothesis of fraud than the divorce-plus-affair scenario. Why not?

Imagine a backstory such that Carson's affair, considered in isolation, supports fraud much more strongly than a trip to Brazil, considered in isolation. Perhaps poor Carson is in over his head: his affair is a surprise to all, and an expensive one at that. His trip to Rio, although unusual, pales by comparison, and on its own there is no reason to think that his having a shady seat companion is anything but a coincidence. Relative to this background knowledge, $A, P_{A}\left(H \mid E_{3}\right)>P_{A}\left(H \mid E_{2}\right)$. Now add the news of Carson's divorce proceedings. This would not dent the confirmatory power of the affair scenario, since adding a divorce to that affair only piles on more expenses for Carson. But, learning of the divorce would also do little to alter the confirmatory force of the travel scenario on judging whether Carson is complicit in defrauding Acme. Thus, $P_{A}\left(H \mid\left\{E_{1}, E_{3}\right\}\right)>P_{A}\left(H \mid\left\{E_{1}, E_{2}\right\}\right)$.

But then there is little reason to believe that we need the coincidence of a divorce and an affair to send up a red flag in Acme's fraud department, as opposed to just knowing of Carson's liaison. The coherence of divorce and affair may therefore be less than the coherence of divorce and travel with respect to confirming the hypothesis of fraud, and still the conjunction of divorce and affair may be more confirmatory of fraud than the conjunction of divorce and travel. As we have seen, this happens when the confirmatory power of the affair (considered by itself) becomes sufficiently large in comparison with the confirmatory power of the travel (considered by itself). When coherence is measured by focused correlation, what qualifies as 'sufficiently large' is then precisely quantified by the bound (2).

\subsection{From confirmation to focused correlation}

Let us now tackle the converse relation, namely, the implication from a difference in confirmation to a difference in focused correlation.

Proposition 3.2. If $c\left(H,\left\{E_{1}, E_{2}\right\}\right)>c\left(H,\left\{E_{1}, E_{3}\right\}\right)$ and

$$
P\left(H \mid E_{2}\right) \leq P\left(H \mid E_{3}\right),
$$

then also $\operatorname{For}_{H}\left(E_{1}, E_{2}\right)>\operatorname{For}_{H}\left(E_{1}, E_{3}\right)$.

The bound (3) is a catch-all condition that holds for all confirmation measures $c(H, \mathbf{E})$ considered in Section 2.1. It may be tightened by focusing on a particular measure. For example, for $i n c_{1}(H, \mathbf{E})$ and $i n c_{2}(H, \mathbf{E})$ the bound is

$$
\frac{P\left(H \mid E_{2}\right)}{P\left(H \mid E_{3}\right)}<\frac{i n c_{k}\left(H, E_{1}, E_{2}\right)}{i n c_{k}\left(H, E_{1}, E_{3}\right)}, \quad k=1,2,
$$


which allows for $P\left(H \mid E_{2}\right)>P\left(H \mid E_{3}\right)$ within certain limits.

The existence of a bound on the ratio of $P\left(H \mid E_{2}\right)$ to $P\left(H \mid E_{3}\right)$ has a similar explanation as the bound (2). If $P\left(H \mid E_{2}\right)>P\left(H \mid E_{3}\right)$ by a sufficiently large amount, then the larger confirmatory power of $\mathbf{E}$ over $\mathbf{E}^{*}$ may be overshadowed by the fact that the confirmation of $H$ by $E_{3}$ alone, with respect to the confirmation by the conjunction $E_{1} \wedge E_{3}$, is much less than the confirmation of $H$ by $E_{2}$ alone, with respect to the confirmation by the conjunction $E_{1} \wedge E_{2}$. Or, put more plainly, the beliefs in the set $\mathbf{E}^{*}$ cohere better towards supporting $H$ than the beliefs in the set $\mathbf{E}$.

Example 2. In the spirit of the Introduction, suppose that Carson's affair is viewed as an ordinary disaster and his travel plans are more suspicious - it is known that he booked both his seat and his companion's, let's say. So, suppose that the conjunction of divorce and travel is more confirmatory of the hypothesis of fraud than the conjunction of divorce and an affair on this background knowledge, labeled $B: c_{B}\left(H,\left\{E_{1}, E_{2}\right\}\right)>$ $c_{B}\left(H,\left\{E_{1}, E_{3}\right\}\right)$ and $P_{B}\left(H \mid E_{1} \wedge E_{2}\right)>P_{B}\left(H \mid E_{1} \wedge E_{3}\right)$.

Now, what happens when we consider focused correlation instead of confirmation? Equation (1) weighs $P_{B}\left(H \mid E_{1} \wedge E_{2}\right)$ and $P_{B}\left(H \mid E_{1} \wedge E_{3}\right)$ by how much each of the individual pieces of evidence differing between the two evidence sets - that is, Carson's travel $\left(E_{2}\right)$ and affair $\left(E_{3}\right)$ - confirm the suspicion of fraud. If condition (3) is violated, i.e., if $P_{B}\left(H \mid E_{2}\right)>P_{B}\left(H \mid E_{3}\right)$, this would mean that isolated knowledge of Carson's travel makes us more inclined to think of the possibility that he may be engaged in fraudulent business than if we simply heard that Carson is embroiled in an affair. It is the merit of focused correlation to now put up a cautionary flag when it comes to considering the implications for the relative degrees of coherence of the two evidence sets $\left\{E_{1}=\right.$ divorce, $E_{2}=$ travel $\}$ and $\left\{E_{1}=\right.$ divorce, $E_{3}=$ affair $\}$. How so?

The argument is similar to the discussion of the previous example in Sec. 3.2. Since according to background $B$ the evidence of travel, considered in isolation, is already more supportive of the hypothesis of fraud than the evidence of an affair, also considered in isolation, and since the only other piece of evidence - namely, the fact that Carson is in the midst of divorce proceedings - is common to both evidence sets, then one should not automatically conclude that it is the coincidence of divorce and travel that boosts our confidence in fraud. Rather, it may be the case that the evidence of travel alone is what makes the hypothesis so eminently plausible, not the fact that it coincides with Carson's divorce. Conversely, while Carson's affair alone may barely raise suspicions of fraud, its coincidence with Carson's being in the midst of a messy - and potentially financially threatening - divorce may boost the likelihood of fraud by a large margin. In other words, although the coincidence of Carson's divorce and travel is more confirmatory of fraud than the coincidence of his divorce and affair, the latter evidence set may well be judged to have a larger degree of coherence. Focused correlation captures this subtlety.

Conversely, if condition (3) is obeyed, i.e., if $P_{B}\left(H \mid E_{2}\right) \leq P_{B}\left(H \mid E_{3}\right)$, focused correlation and confirmation will gravitate toward favoring the same evidence set. Together with the fact that $P_{B}\left(H \mid E_{1} \wedge E_{2}\right)>P_{B}\left(H \mid E_{1} \wedge E_{3}\right)$, we can now safely conclude that divorce and travel cohere better toward supporting the hypothesis of fraud than the 
conjunction of divorce and affair: although evidence of travel alone is less (or equally) indicative of fraud compared with the evidence of an affair, when taken together with the evidence of an ongoing divorce, Carson's travel will ring Acme's alarm bells more readily than evidence of Carson's involvement with his Brazilian sweetheart.

\subsection{Bidirectional tracking}

Propositions 3.1 and 3.2 show that when the ratio of $P\left(H \mid E_{2}\right)$ to $P\left(H \mid E_{3}\right)$ is within a certain range, focused correlation will be tracked by confirmation, and when it is within another range, focused correlation will track confirmation. It is important to note that these ranges overlap. Propositions 3.1 and 3.2 readily quantify this overlap, i.e., the range of values of $P\left(H \mid E_{2}\right) / P\left(H \mid E_{3}\right)$, for which bidirectional tracking holds. Combining Propositions 3.1 and 3.2 yields:

Corollary 3.1. If

$$
\frac{\operatorname{For}_{H}\left(E_{1}, E_{3}\right)}{\operatorname{For}_{H}\left(E_{1}, E_{2}\right)}<\frac{P\left(H \mid E_{2}\right)}{P\left(H \mid E_{3}\right)} \leq 1,
$$

then $\operatorname{For}_{H}\left(E_{1}, E_{2}\right)>\operatorname{For}_{H}\left(E_{1}, E_{3}\right) \Leftrightarrow c\left(H,\left\{E_{1}, E_{2}\right\}\right)>c\left(H,\left\{E_{1}, E_{3}\right\}\right)$.

That is, ensuring that the tracking goes both ways does not require us to impose the rather strong assumption (A2) of equal relevance of all bits of evidence.

Choosing a particular confirmation measure allows us to extend even further the range over which the bidirectional tracking holds. For example, for $i_{n} c_{1}(H, \mathbf{E})$ or $i n c_{2}(H, \mathbf{E})$, we can use the bound (4) instead of (3), which gives a 'relaxed' version of Corollary 3.1:

\section{Corollary 3.2. If}

$$
\frac{\operatorname{For}_{H}\left(E_{1}, E_{3}\right)}{\operatorname{For}_{H}\left(E_{1}, E_{2}\right)}<\frac{P\left(H \mid E_{2}\right)}{P\left(H \mid E_{3}\right)}<\frac{i n c_{k}\left(H, E_{1}, E_{2}\right)}{i n c_{k}\left(H, E_{1}, E_{3}\right)}, \quad k=1,2,
$$

then $\operatorname{For}_{H}\left(E_{1}, E_{2}\right)>\operatorname{For}_{H}\left(E_{1}, E_{3}\right) \Leftrightarrow \operatorname{inc}_{k}\left(H,\left\{E_{1}, E_{2}\right\}\right)>\operatorname{inc}_{k}\left(H,\left\{E_{1}, E_{3}\right\}\right)$.

Fig. 1 illustrates the different ranges of $P\left(H \mid E_{2}\right) / P\left(H \mid E_{3}\right)$ for which uni- and bidirectional tracking between focused correlation and confirmation obtains.

\subsection{Equality of focused correlation and confirmation}

For the sake of completeness, let us also explicitly consider the connection between equal values of focused correlation and equal values of confirmation:

Proposition 3.3. For $_{H}\left(E_{1}, E_{2}\right)=$ For $_{H}\left(E_{1}, E_{3}\right) \Leftrightarrow c\left(H,\left\{E_{1}, E_{2}\right\}\right)=c\left(H,\left\{E_{1}, E_{3}\right\}\right)$ if and only if

$$
P\left(H \mid E_{2}\right)=P\left(H \mid E_{3}\right) .
$$

In other words, equal values of focused correlation for the two sets $\mathbf{E}$ and $\mathbf{E}^{*}$ translate into equal degrees $c\left(H,\left\{E_{1}, E_{2}\right\}\right)$ and $c\left(H,\left\{E_{1}, E_{3}\right\}\right)$ of confirmation-and vice versaif and only if the equal-relevance assumption (A2) is made. The reason for this result should now be clear from the above discussion of Propositions 3.1 and 3.2. 


$$
c \Rightarrow \text { For }
$$
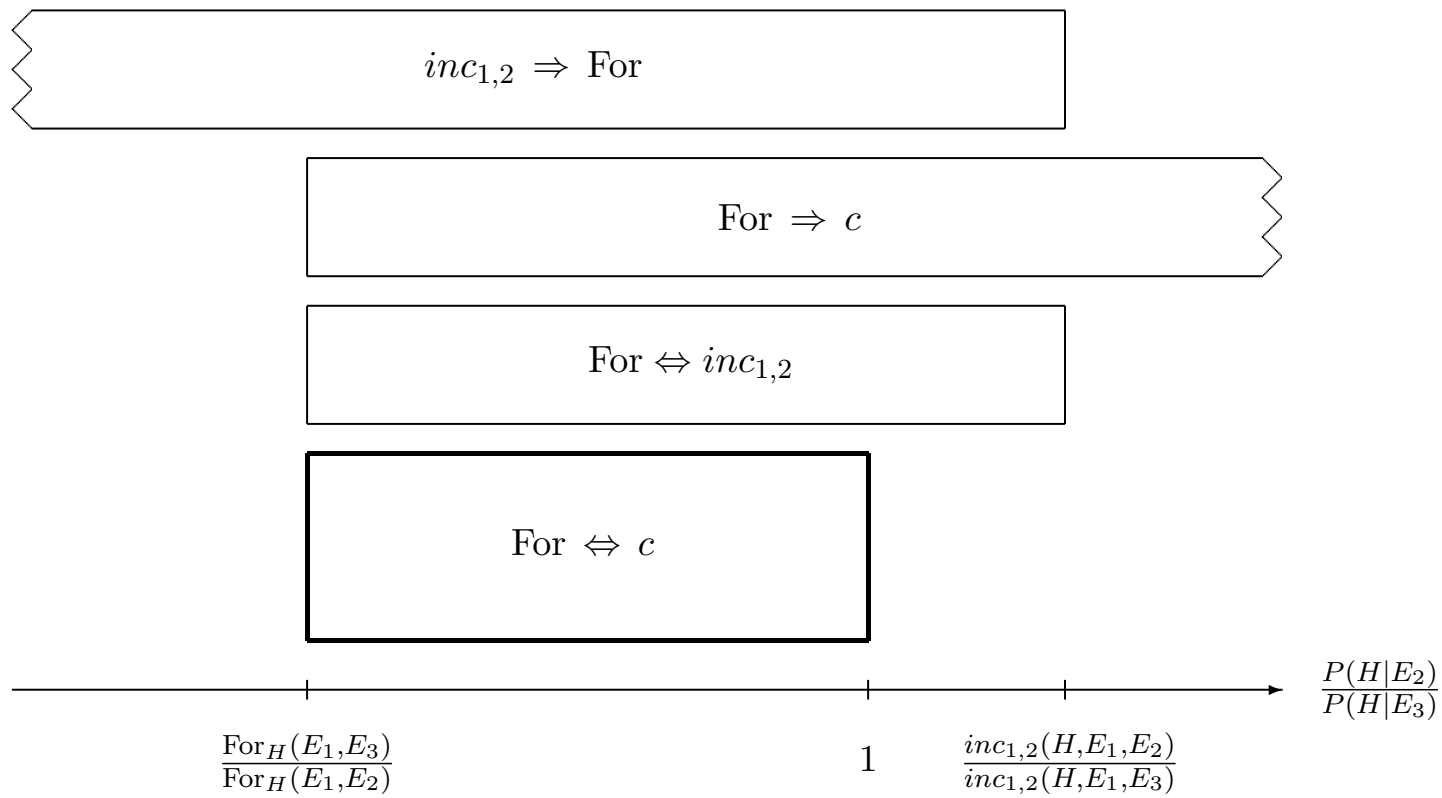

Figure 1: Regimes of $P\left(H \mid E_{2}\right) / P\left(H \mid E_{3}\right)$ for which focused correlation tracks and/or is tracked by confirmation measures. $P\left(H \mid E_{i}\right)$ is the relevance of a piece of evidence $E_{i}$ for a given hypothesis $H$. 'For $\Rightarrow c$ ' is a shorthand for the implication $\operatorname{For}_{H}\left(E_{1}, E_{2}\right)>\operatorname{For}_{H}\left(E_{1}, E_{3}\right) \Rightarrow$ $c\left(H,\left\{E_{1}, E_{2}\right\}\right)>c\left(H,\left\{E_{1}, E_{3}\right\}\right)$, which represents a tracking of focused correlation by the confirmation measures $c(H, \mathbf{E})$. Similarly for ' $c \Rightarrow$ For.' Over a range of values for $P\left(H \mid E_{2}\right) / P\left(H \mid E_{3}\right)$, bidirectional tracking obtains (bold box), i.e., $\operatorname{For}_{H}\left(E_{1}, E_{2}\right)>\operatorname{For}_{H}\left(E_{1}, E_{3}\right) \Leftrightarrow$ $c\left(H,\left\{E_{1}, E_{2}\right\}\right)>c\left(H,\left\{E_{1}, E_{3}\right\}\right)$. For specific choices of $c(H, \mathbf{E})$, for example, for the measures $i n c_{1}(H, \mathbf{E})$ or $i n c_{2}(H, \mathbf{E})$ shown here, the tracking range is extended further. 


\section{Discussion}

In this paper, we have demonstrated that the equal-relevance assumption can be relaxed within certain limits without upsetting the bidirectional tracking between focused correlation and several incremental confirmation measure; if only unidirectional tracking is required, these limits become even less stringent. However, if the degrees of relevance diverge too strongly, the tracking fails. We have shown that the ratio of $P\left(H \mid E_{2}\right)$ to $P\left(H \mid E_{3}\right)$ is crucial in assessing, both qualitatively and quantitatively, when and where the tracking fails.

However, this observation should not be viewed as reflecting a failure or deficiency of the measure of focused correlation. Rather, it may be considered as illuminating a shortcoming of incremental confirmation measures, since they fail to take into account the additional information provided by the relevance of each individual bit of evidence. As we have seen, this information is important in flagging a situation in which a large boost in confirmation caused by adding a piece of evidence has nothing or only little to do with the coincidence of this evidence with the existing body of evidence. One merit of focused correlation is to detect such cases.

In one class of cases that has received a lot of attention, namely, the single-factor common-cause model mentioned in the Introduction, any measure linking positive association to positive confirmation breaks down, and comparisons (assuming (A2)) are never false because they cannot be different. But these facts take nothing away from focused correlation, for two reasons. First, because this class of models is determined non-parametrically, we can learn whether one hypothesis is a common cause (Silva et al. 2006) and then attack the problem by other methods - namely by looking at the relative strengths of the evidence. We don't need the sign of focused correlation in single-factor common-cause models to indicate the sign of confirmation if we understand clearly when the two do not align. In the case of single-factor common-cause models, the association of the evidence is completely determined by the relevant strengths of the evidence (Danks and Glymour 2001). Faulting focused correlation for failing to indicate confirmation in this class of models is akin to faulting screwdrivers for failing to drive nails: it is a true claim that is entirely beside the point. Second, it should be clear that the failure of focused correlation to track differences in confirmation of evidence in common-cause models in Proposition 2.1 is driven by (A2) rather than a deficiency in focused correlation. But, again, although our results here allow for some tracking in common-cause models, at bottom a difference in confirmation in this class of models boils down to a difference in the individual strengths, $P\left(H \mid E_{i}\right)$.

Focused correlation is defined generally for $n$ evidence variables, but we only discuss evidence sets of size 2, and difficulties loom for attempts to make comparisons of larger evidence sets (Bovens and Hartmann 2006). Even though focused correlation is defined for arbitrary-sized information sets, the relationships of confirmation, covariance, and correlation are binary - or three-place in conditional form. Thus, the expansion of evidence sets beyond size two will require a partition of the evidence set, since there are many incremental confirmation questions that are compatible with one focused corre- 
lation problem involving an evidence set of size greater than two. To expect otherwise is a category mistake, and negative results should be no surprise. That said, if there isn't an interest in linking focused correlation for large evidence sets - which, technically, isn't a measure of correlation but is instead a distance-from-independence measure - to confirmation, then one may explore extending the results presented here to facilitate direct comparisons of large evidence sets.

Finally, two commonly discussed confirmation measures have been omitted from discussion, Carnap's (1962) relevance measure $\mathfrak{r}$, and the old evidence measure oe (Christensen 1999, Joyce 1999), which are defined as

$$
\begin{aligned}
\mathfrak{r}(H, E) & :=P(H \wedge E)-P(H) P(E), \\
o e(H, E) & :=P(H \mid E)-P(H \mid \neg E) .
\end{aligned}
$$

Neither measure is tracked by focused correlation. The reason is that both measures are less constrained than incremental confirmation measures. In the case of Carnap's measure of relevance, observe that $\mathfrak{r}(H, E)$ is the covariance of the binary variables $H$ and $E$,

$$
\operatorname{Cov}(H, E)=P(H \wedge E)-P(H) P(E)=P(H)(P(E \mid H)-P(E)) .
$$

Although positive covariance for $H$ and a single evidence variable $E$ is ensured by the positive relevance condition (A1), conditions (A1) and (A2) and Equation (9a) do not constrain the sign of $\mathfrak{r}(H, \mathbf{E})$ for evidence sets $\mathbf{E}$ of size two. In the case of oe, there is some recognition already of the difference between incremental confirmation measures and measures for novelty of evidence like oe (Joyce 1999). The failure to compare evidence sets by their degree of surprise, particularly under the restrictive conditions of positivity, (A1), (A2), and Equation (9a), is an additional reason to sharply distinguish between these two types of measures. Counter-models for oe are discussed in the Appendix.

\section{References}

Bovens, L. and Hartmann, S. (2003). Bayesian Epistemology. Oxford University Press, Oxford.

Bovens, L. and Hartmann, S. (2006). An impossibility result for coherence rankings. Philosophical Studies, 128:77-91.

Carnap, R. (1962). The Logical Foundations of Probability. University of Chicago Press, 2nd edition.

Christensen, D. (1999). Measuring confirmation. Journal of Philosophy, 96:437-461.

Cohen, L. J. (1977). The Probable and the Provable. Clarendon Press, Oxford. 
Crupi, V., Tentori, K., and Gonzalez, M. (2007). On Bayesian measures of evidential support: Theoretical and empirical issues. Philosophy of Science, 74(2):229-252.

Danks, D. and Glymour, C. (2001). Linearity properties of Bayes nets with binary variables. In Breese, J. and Koller, D., editors, Uncertainty in Artificial Intelligence: Proceedings of the 17th conference (UAI-2001), pages 88-104.

Eells, E. and Fitelson, B. (2002). Symmetries and asymmetries in evidential support. Philosophical Studies, 107(2):129-142.

Joyce, J. (1999). The Foundations of Causal Decision Theory. Cambridge University Press, Cambridge.

Kemeny, J. and Oppenheim, P. (1952). Degrees of factual support. Philosophy of Science, 19:307-324.

Keynes, J. M. (1921). A Treatise on Probability. Macmillan, London.

Kyburg, Jr., H. E. (1983). Recent work in inductive logic. In Machan, T. and Lucey, K., editors, Recent Work in Philosophy, pages 87-150. Rowman and Allanheld.

Milne, P. (1997). $\log [p(h \mid e b) / p(h / b)]$ is the one true measure of confirmation. Philosophy of Science, 63:21-26.

Myrvold, W. (1996). Bayesianism and diverse evidence: A reply to Andrew Wayne. Philosophy of Science, 63:661-665.

Myrvold, W. (2003). A Bayesian account of the virtue of unification. Philosophy of Science, 70:399-423.

Olsson, E. (2005). Against Coherence: Truth, Probability and Justification. Oxford University Press, Oxford.

Silva, R., Glymour, C., Scheines, R., and Spirtes, P. (2006). Learning the structure of latent linear structure models. Journal of Machine Learning Research, 7:191-246.

Wheeler, G. (2009). Focused correlation and confirmation. The British Journal for the Philosophy of Science, 60(1):79-100.

Wheeler, G. and Scheines, R. (2010). Causation, association, and confirmation. In Explanation, Prediction, and Confirmation: New Trends and Olds Ones Reconsidered, The Philosophy of Science in a European Perspective. Springer. 


\section{Appendix}

The following proofs of Propositions 3.1-3.3 are similar in spirit and rely heavily on the following Lemma 4.1, which follows from the definition of $\operatorname{For}_{H}\left(E_{1}, E_{2}\right)$ in Equation (1).

Lemma 4.1. If For $_{H}\left(E_{1}, E_{2}\right)=\lambda$ For $_{H}\left(E_{1}, E_{3}\right)$ with $\lambda>0$, then

$$
\frac{P\left(H \mid E_{1} \wedge E_{2}\right)}{P\left(H \mid E_{1} \wedge E_{3}\right)}=\lambda \frac{P\left(H \mid E_{2}\right)}{P\left(H \mid E_{3}\right)} .
$$

\section{Proof of Lemma 4.1}

$$
\begin{aligned}
\frac{P\left(H \mid E_{1} \wedge E_{2}\right) P(H)}{P\left(H \mid E_{1}\right) P\left(H \mid E_{2}\right)} & =\lambda \frac{P\left(H \mid E_{1} \wedge E_{3}\right) P(H)}{P\left(H \mid E_{1}\right) P\left(H \mid E_{3}\right)} \\
\frac{P\left(H \mid E_{1} \wedge E_{2}\right)}{P\left(H \mid E_{2}\right)} \times \frac{P(H)}{P\left(H \mid E_{1}\right)} & =\lambda \frac{P\left(H \mid E_{1} \wedge E_{3}\right)}{P\left(H \mid E_{3}\right)} \times \frac{P(H)}{P\left(H \mid E_{1}\right)} \\
\frac{P\left(H \mid E_{1} \wedge E_{2}\right)}{P\left(H \mid E_{1} \wedge E_{3}\right)} & =\lambda \frac{P\left(H \mid E_{2}\right)}{P\left(H \mid E_{3}\right)} .
\end{aligned}
$$

\section{Proof of Proposition 3.1}

We begin with two observations, assuming (A1) and (A2):

$$
\begin{aligned}
& P\left(H \mid E_{1} \wedge E_{2}\right)>P\left(H \mid E_{1} \wedge E_{3}\right) \Leftrightarrow c\left(H,\left\{E_{1}, E_{2}\right\}\right)>c\left(H,\left\{E_{1}, E_{3}\right\}\right), \\
& P\left(H \mid E_{1} \wedge E_{2}\right)=P\left(H \mid E_{1} \wedge E_{3}\right) \Leftrightarrow c\left(H,\left\{E_{1}, E_{2}\right\}\right)=c\left(H,\left\{E_{1}, E_{3}\right\}\right) .
\end{aligned}
$$

Turning to $\operatorname{For}_{H}(\mathbf{E})$, by (A2), $P\left(H \mid E_{2}\right) / P\left(H \mid E_{3}\right)=1$. So, by Lemma 4.1, the LHS of Equations (9a) and (9b) may be expressed by Equation (10) when $\lambda>1$ and $\lambda=1$, respectively.

$$
\frac{P\left(H \mid E_{1} \wedge E_{2}\right)}{P\left(H \mid E_{1} \wedge E_{3}\right)}=\lambda .
$$

Without (A2), observe:

$$
\begin{aligned}
\frac{P\left(H \mid E_{1} \wedge E_{2}\right)}{P\left(H \mid E_{1} \wedge E_{3}\right)} & =\lambda \frac{P\left(H \mid E_{2}\right)}{P\left(H \mid E_{3}\right)}, \\
\frac{P\left(H \mid E_{1} \wedge E_{2}\right)}{P\left(H \mid E_{1} \wedge E_{3}\right)} \times \frac{P\left(H \mid E_{3}\right)}{P\left(H \mid E_{2}\right)} & =\lambda, \\
\frac{P\left(H \mid E_{1} \wedge E_{2}\right) P(H)}{P\left(H \mid E_{1}\right) P\left(H \mid E_{2}\right)} \times \frac{P\left(H \mid E_{1}\right) P\left(H \mid E_{3}\right)}{P\left(H \mid E_{1} \wedge E_{3}\right) P(H)} & =\lambda, \\
\frac{\operatorname{For}_{H}\left(E_{1}, E_{2}\right)}{\operatorname{For}_{H}\left(E_{1}, E_{3}\right)} & =\lambda .
\end{aligned}
$$


So $\lambda>1$, given (11) and $\operatorname{For}_{H}\left(E_{1}, E_{2}\right)>\operatorname{For}_{H}\left(E_{1}, E_{3}\right)$, by hypothesis. Therefore, $P\left(H \mid E_{1} \wedge E_{2}\right)>P\left(H \mid E_{1} \wedge E_{3}\right)$ and thus $c\left(H,\left\{E_{1}, E_{2}\right\}\right)>c\left(H,\left\{E_{1}, E_{3}\right\}\right)$ iff

$$
\frac{P\left(H \mid E_{2}\right)}{P\left(H \mid E_{3}\right)}>\frac{1}{\lambda}=\frac{\operatorname{For}_{H}\left(E_{1}, E_{3}\right)}{\operatorname{For}_{H}\left(E_{1}, E_{2}\right)} .
$$

\section{Proof of Proposition 3.2}

By assumption, $c\left(H,\left\{E_{1}, E_{2}\right\}\right)>c\left(H,\left\{E_{1}, E_{3}\right\}\right)$, and thus $P\left(H \mid E_{1} \wedge E_{2}\right)>P(H \mid$ $\left.E_{1} \wedge E_{3}\right)$ from Equations (9a) and (9b). Then by Lemma 4.1

$$
\begin{aligned}
& \frac{P\left(H \mid E_{1} \wedge E_{2}\right)}{P\left(H \mid E_{1} \wedge E_{3}\right)}>\frac{P\left(H \mid E_{2}\right)}{P\left(H \mid E_{3}\right)}, \\
& \frac{P\left(H \mid E_{1} \wedge E_{2}\right)}{P\left(H \mid E_{2}\right)}>\frac{P\left(H \mid E_{1} \wedge E_{3}\right)}{P\left(H \mid E_{3}\right)} .
\end{aligned}
$$

We can tighten the bound if we choose a particular confirmation measure. For example,

$$
\begin{aligned}
\quad \operatorname{inc}_{k}\left(H, E_{1}, E_{2}\right) & =\gamma i n c_{k}\left(H, E_{1}, E_{3}\right) \\
\Leftrightarrow \quad P\left(H \mid E_{1} \wedge E_{2}\right) & =\gamma P\left(H \mid E_{1} \wedge E_{3}\right), \quad k=1,2,
\end{aligned}
$$

and thus the bound is

$$
\frac{P\left(H \mid E_{2}\right)}{P\left(H \mid E_{3}\right)}<\gamma=\frac{i n c_{k}\left(H, E_{1}, E_{2}\right)}{i n c_{k}\left(H, E_{1}, E_{3}\right)}
$$

which proves Equation (4).

\section{Proof of Proposition 3.3}

If $\operatorname{For}_{H}\left(E_{1}, E_{2}\right)=\operatorname{For}_{H}\left(E_{1}, E_{3}\right)$, then Definition (1) implies that $P\left(H \mid E_{1} \wedge E_{2}\right)=P(H \mid$ $\left.E_{1} \wedge E_{3}\right)$ iff $P\left(H \mid E_{2}\right)=P\left(H \mid E_{3}\right)$. Conversely, if $c\left(H,\left\{E_{1}, E_{2}\right\}\right)=c\left(H,\left\{E_{1}, E_{3}\right\}\right)$, then Equation (9b) implies that $P\left(H \mid E_{1} \wedge E_{2}\right)=P\left(H \mid E_{1} \wedge E_{3}\right)$, and Definition (1) then shows that $\operatorname{For}_{H}\left(E_{1}, E_{2}\right)=$ For $_{H}\left(E_{1}, E_{3}\right)$ iff $P\left(H \mid E_{2}\right)=P\left(H \mid E_{3}\right)$.

\section{Counter-models for measure tracking oe}

To show that $P\left(H \mid E_{1} \wedge E_{2}\right)>P\left(H \mid E_{1} \wedge E_{3}\right)$ does not entail oe $\left(H,\left\{E_{1}, E_{2}\right\}\right)>$ $o e\left(H,\left\{E_{1}, E_{3}\right\}\right)$ under positivity, (A1) and (A2), it suffices to provide a model in which

$$
P\left(H \mid E_{1} \wedge E_{2}\right)>P\left(H \mid E_{1} \wedge E_{3}\right)
$$

but

$$
P\left(H \mid E_{1} \wedge E_{2}\right)-P\left(H \mid \neg E_{1} \vee \neg E_{2}\right) \ngtr P\left(H \mid E_{1} \wedge E_{3}\right)-P\left(H \mid \neg E_{1} \vee \neg E_{3}\right) .
$$

The constraints (A1) and (A2) are strong by design, and we can simplify Equation (15) by observing the following Lemma. 
Lemma 4.2. If $P(\mathbf{D})$ is a positive probability distribution over $\langle H, \mathbf{E}\rangle$ satisfying (A1) and (A2), then

i. $P\left(E_{1}\right)=P\left(E_{2}\right)=P\left(E_{3}\right)$, and

ii. $P\left(H \mid \neg E_{1}\right)=P\left(H \mid \neg E_{2}\right)=P\left(H \mid \neg E_{3}\right)=\alpha$.

\section{Proof of Lemma 4.2}

4.2.i.: From (A2), it follows that the covariances of each piece of evidence with the hypothesis is identical, $P\left(E_{i} \mid H\right) / P\left(E_{i}\right)$ for $i=1,2,3$, which can be rewritten as

$$
P\left(E_{i}\right)\left[P\left(H \mid E_{i}\right)-P(H)\right] .
$$

(A2) guarantees 4.2.i., and (A1) ensures that the covariance is positive.

4.2.ii.: From total probability, (A2) and Lemma 4.2.i.:

$$
P(H)=P\left(H \mid E_{i}\right) P\left(E_{i}\right)+P\left(H \mid \neg E_{i}\right) 1-P\left(E_{i}\right) .
$$

Lemma 4.2 allows for the reduction of (15) to

$$
\begin{aligned}
P\left(H \mid E_{1} \wedge E_{2}\right)-\left[2 \alpha-P\left(H \mid \neg E_{1} \wedge \neg E_{2}\right)\right] & \\
& \ngtr \\
& P\left(H \mid E_{1} \wedge E_{3}\right)-\left[2 \alpha-P\left(H \mid \neg E_{1} \wedge \neg E_{3}\right)\right] .
\end{aligned}
$$

But while $P\left(H \mid E_{1} \wedge E_{2}\right)>P\left(H \mid E_{1} \wedge E_{3}\right)$ by hypothesis, the remaining terms are unconstrained. Counter-models exist which violate the inequality. Analogously, there are counter models in which $P\left(H \mid E_{1} \wedge E_{2}\right)=P\left(H \mid E_{1} \wedge E_{3}\right)$ but oe $\left(H, E_{1} \wedge E_{2}\right) \neq$ oe $\left(E_{1} \wedge E_{3}\right)$.

In sum, more incremental confirmation does not ensure more evidential surprise, and it should be clear from the discussion that the converse does not hold either. 